\section{EL TRICORNIO DE LA GUARDIA CIVIL COMO SÍMBOLO LITERARIO EN LOS RELATOS DE VIAJEROS DE HABLA INGLESA DEL SIGLO XX}

\author{
José Ruiz Mas \\ Departamento de Filologías Inglesa y Alemana \\ Universidad de Granada \\ jrmas@ugr.es
}

Cómo citar este artículo/ Citation: Ruiz Mas, J. (2013). El tricornio de la Guardia Civil como símbolo literario en los relatos de viajeros de habla inglesa del siglo XX. Arbor, 189(760):a024. doi: http://dx.doi.org/10.3989/arbor.2013.760n2010

Recibido: 15 diciembre 2012; Aceptado: 24 enero 2013

RESUMEN: La presencia en la literatura en lengua inglesa del sombrero de la Guardia Civil, más conocido popularmente como 'tricornio', empezó mediante una mera descripción visual de una prenda difícil de 'aprehender' ('three-cornered hat' o 'cocked hat') y se fue paulatinamente cargando de significado y matices, variables según la época y el viajero, sobre todo durante el siglo XX. Se fue complementando con el tiempo con variable adjetivación, numerosas impresiones personales del viajero e interpretaciones más o menos rocambolescas sobre su origen o uso: referencias a su utilidad para permitir a los guardias pegarse a la pared y así atrapar a los malhechores; su escaso valor como protector de la lluvia pero su utilidad para proteger las orejas de su portador; su vinculación con la guerra de Independencia, con Napoleón o Goya, con la Gendarmería francesa; su arcaísmo; su parecido a los gorros de los monigotes de feria u operetas, etc. La presencia constante del 'tricornio' en el paisaje literario español según los viajeros anglófonos resulta valiosa para entender la España contemporánea.

PALABRAS CLAVE: Guardia Civil; sombrero; relatos de viajeros de habla inglesa en España; sombrero de tres picos/ tricornio; uniformes militares; charol.

\section{THE CIVIL GUARD'S TRICORN AS A LITERARY SYMBOL IN 2OTH-CENTURY ENGLISH- SPEAKING TRAVELLERS' ACCOUNTS}

Copyright: (C) 2013 CSIC. Este es un artículo de acceso abierto distribuido bajo los términos de la licencia Creative Commons Attribution-Non Commercial (by-nc) Spain 3.0.

ABSTRACT: This presence in English literary texts of the hat worn by the Spanish Guardia Civil, known in Spanish as the 'tricornio', had its beginnings in the form of mere descriptions. It began as an item of clothing that was difficult to 'apprehend' (a 'three-cornered hat' or a 'cocked hat'), but these visual perceptions gradually started to take on subtle shades of meaning and a significance which varied with the historical circumstances and the individual travellers, especially during the 20th century. Its descriptions were complemented in time with varying adjectives, personal impressions and more or less bizarre interpretations as to its origin and purpose, namely: its usefulness in allowing the civil guards to lean against the wall and therefore catch criminals more easily; its poor role as protector against the rain but its success as a protector of the civil guard's ears; its connection with the Peninsular War, Napoleon or Goya, or to the French Gendarmerie; its archaism; its similarity to the hats of funfair puppets and comic operas, etc. Indeed, the constant presence of the 'cocked hat' in the Spanish literary landscape in the eyes of Anglophone travellers is of the utmost relevance when endeavouring to understand contemporary Spain.

KEYWORDS: Guardia Civil; headgear; English-speaking travel accounts on Spain; three-cornered hat/ 'cocked hat'; military uniforms; patent-leather. 
Son escasos los viajeros de habla inglesa que al visitar España con posterioridad a 1844 no hayan dedicado como mínimo unas líneas a una institución tan 'española' como la Guardia Civil. Con frecuencia la Benemérita ha recibido incluso pasajes enteros en los relatos de viajeros anglófonos, y no es tampoco infrecuente que se dedicara incluso algún que otro capítulo a ella en exclusividad, a veces en términos elogiosos, en ocasiones con espíritu abiertamente crítico. Las opiniones sobre la Guardia Civil han venido determinadas en la mayoría de las ocasiones por factores tales como los prejuicios personales, nacionales o ideológicos del viajero, por su clase social, por su economía particular o por su conocimiento de la lengua y la cultura española, entre otras posibles variables que habría que analizar por épocas y viajero por viajero (Ruiz Mas, 2005 y 2010). El Instituto armado, aparte de ser una de las escasísimas instituciones (si no la única) que no ha desaparecido en ningún momento de la turbulenta historia de España desde que fuera creada en $1844,{ }^{1}$ es además una de las pocas que, teniendo su origen en el siglo XIX, aún permanecen en la España del siglo XXI, sabiendo adaptarse a la época, sin perder un ápice de su categoría profesional y lealtad a los gobiernos y a los distintos vaivenes políticos e históricos del país. No en vano es una de las instituciones más valoradas por los españoles.

Si hay algún elemento visual que representa a la Guardia Civil, la cristaliza en la imagen colectiva tanto nacional como internacional y la hace claramente distintiva de otros cuerpos militares y no militares y tipos recurrentes de la España de los dos últimos siglos es su peculiar sombrero, el popularmente conocido como 'tricornio'. Mas, si bien ha sido el elemento dominante que la ha definido, tampoco ha sido el único, como tendremos ocasión de comprobar.

Resulta conveniente dejar bien definido el término y el concepto de relato de viajes, género en el que se percibe con mayor claridad que en ningún otro los rasgos definitorios de un símbolo literario como el sombrero de la Guardia Civil, objeto aquí de mi estudio. Gracias a los relatos de viajes podemos dibujar una imagen concreta de un elemento casi omnipresente del paisaje español como es el tricornio, y lo que es más, podemos percibir la gradual evolución de los diversos rasgos, matices y peculiaridades que han venido dando forma literaria a una realidad visual llamativa a ojos foráneos, para lo cual, qué duda cabe, han influido las diferentes coordenadas históricas, sociales, culturales y políticas que han contextualizado las diversas narraciones de los viajeros del siglo XX, la consiguiente percepción del objeto a describir y la expresión literaria del mismo, en el que han intervenido con frecuencia la lectura de otros relatos de viajes anteriores. Los relatos de viajes, según Alburquerque-García (2011:16-19), responden a tres rasgos fundamentales. En primer lugar, son relatos factuales, es decir, relatos basados en hechos reales (que es en esencia el viaje en sí realizado en un tiempo y un espacio vividos por el viajero), aunque se permite en ellos un cierto grado de ficcionalización (16-17). En segundo lugar se caracterizan por el predominio de la descripción sobre la narración, o lo que es igual, las representaciones de objetos y personajes constituyen el núcleo de la descripción hasta el punto de apoderarse del protagonismo de la obra (17). Y en tercer lugar, se percibe en ellos el mantenimiento de un cierto equilibrio entre la objetividad en la descripción de la experiencia vivida y la parcialidad casi inevitable del autor (el propio viajero) a la hora de poner sobre el papel su testimonio, que aunque tendente a la objetividad, a partir sobre todo del siglo XIX, se inclina también en cierto grado hacia la subjetividad (18).

El uniforme de la Guardia Civil se convierte para los viajeros anglófonos conforme avanza inexorable el siglo XX en una de las señas de identidad visuales claves de la imagen literaria del guardia civil, sobre todo por su característico sombrero, que sigue siendo la prenda que recibe la mayor atención, hasta el punto que para algunos el Instituto armado queda suficientemente descrita mediante una única pincelada. Bien entrados en el siglo XX, en pleno reinado de Alfonso XIII, tanto James R. McClymont, autor de A Scot in Spain (1921), como el teniente coronel H. A. Newell, autor de Footprints in Spain (1922), ambos viajeros posteriores a la Reforma del vestuario de 1909, se aferran a la idea de que el atuendo del guardia civil es anticuado, grotesco y más propio de espectáculo cómico o de opereta que de uniforme militar, sobre todo en lo que respecta al sombrero y a las polainas. El escocés dice ver pasar a soldados 'wearing their early nineteenth century hats and their antiquated gaiters' (1921:6). Newell (1922:13) describe el uniforme de la Guardia Civil con la objetividad y la profesionalidad propia del militar que es, pero no puede evitar añadir al final que 'their hats are extremely curious, almost grotesque, with a decided suggestion of comic opera' (13). Miss Alice C. D. Riley (Skimming Spain in Five Weeks by Motor, 1931) describe la uniformidad del guardia con un simple 'elegant uniform and patent-leather hat', prenda que solo unas líneas más abajo, curiosamente, califica de 'absurd' (1931:11). Sir Harry Johnston y Dr. L. Haden Guest, autores de The Outline of the World To-day (c.1930), lo califican de 'melodramatic cocked hat' (c.1930, I:228). Con tal prenda los guardias civiles, siguen diciendo, dan una imagen exagerada de ley y de orden (l:228). Para el Gerald Brenan de South from Granada (1957), los 'winged patentleather hats that shine so brightly in the sun' (1957:228) recuerdan a las alas de Cupido, mensajero de la diosa Venus, bajo cuyo signo nacen las dictaduras. ${ }^{2}$ Mediante tan mitológica alusión Brenan aprovecha el emblemático sombrero del guardia civil para señalar la afinidad de la Benemérita con los regímenes dictatoriales de los generales Primo de Rivera y Franco, los cuales él conoció de primera mano en sus estancias españolas. Para C. P. 
Hawkes, autor de Mauresques (1926), la aparición nocturna de 'Guardias Civiles with bright tricorne hats' les hace semejarse a siniestras aves al acecho (1926:167). Sobre esta imagen de pájaro de mal agüero que le proporciona al guardia civil tan particular sombrero se extiende también Henry Albert Phillips (Meet the Spaniards, 1931): 'black crows' les llama (1931:80). Añade además una referencia al armamento de la Guardia Civil ('villanous-looking carbine') y a otros elementos característicos de su vestuario como las 'long olive-gray capes with maroon trimmings':

We see our first guardia civil, always to be found in duplicate, like the carbinieri (sic) of Italy, though not so ornate in their black patent-leather hats with brims unexpectedly turned up in front, suggesting their sobriquet of 'black crows', each armed with a short, villainous-looking carbine. (80)

El hecho de que Thomas Ewing Moore califique en In the Heart of Spain (1927) el sombrero del guardia civil de 'curious and impracticable' no le impide señalar a la Guardia Civil como el cuerpo de uniforme más elegante de los existentes en Andalucía (1927:69). Moore no puede resistir la tentación de describir en qué consiste ese 'curioso' e 'impracticable' sombrero que él denomina 'head-covering' así como de señalar su mayor desventaja: el de estar doblado hacia arriba en su parte delantera, por lo que no resguarda la cara de la lluvia, permitiendo que ésta caiga por el cogote (69). Esta afirmación resulta tener fortuna entre los viajeros de habla inglesa de la época. En efecto, el comentario sobre el tipo de protección que ofrecen las alas del sombrero a las orejas del guardia civil la repiten otros viajeros, como demuestra la conversación que mantiene Miss Riley (1931:11) con su compañera de viaje. En Tarifa un guardia civil se ofrece para mostrarles el camino a las murallas de la ciudad. La escritora pregunta discretamente a su amiga el porqué de tan absurdos sombreros, especialmente con el calor que hace. Y además, de charol, añade. La respuesta: para que no se mojen las orejas cuando llueve (11).

El uniforme de diario toma a partir del reglamento de 1921 , vigente hasta 1931, un color gris-verde del que nos da noticia el capitán Leslie Richardson en Things Seen in the Pyrenees, French and Spanish (1928:136). Pero las referencias al color verde de un uniforme que resulta ya mucho menos vistoso que en épocas anteriores son aún tímidas. Incluso a ojos de Vernon Howe Bailey (Little Known Towns from Spain, c.1928) el uniforme de diario no es verde sino gris (c.1928:55). Richardson (1928:136) prefiere dejar constancia literaria del uniforme de gala del guardia civil, mucho más impactante a ojos del anglosajón, a pesar de ser mucho menos frecuente que el de diario. El aferrarse al vestuario de gala de la Guardia Civil le permite utilizar con propiedad un calificativo como 'picturesque' destinado a venir a menos en los libros de viajes posteriores:
Their workday uniform is a kind of green, but on 'gala' days they wear a black tunic with red and yellow facings, white breeches, and a three-cornered hat. This on week days, in common with the headdress of all Spanish uniforms, is covered with a black waterproof cover. 'La Guardia Civil' are the only police with a sense of the picturesque: they lend romance alike to river or mountain. (136)

También encuentra Charles L. Freeston más vistosa la anticuada e infrecuente uniformidad de gala que la de diario en su popular The Roads of Spain (1930). Menciona las capas verdes - a todas luces un error, pues eran entonces de un color azul oscuro cercano al negro- y los relucientes sombreros que portaban durante el servicio diario (1930:105). Para Freeston, incluir en su relato la descripción del uniforme de día festivo, repleto de colorido, es una tentación irresistible: son, dice, 'brilliant uniforms of red, white, and blue' (105).

Vernon Howe Bailey ofrece en Little Well Known Towns of Spain (c.1928) una descripción seria y fiel del verdadero uniforme de diario de la Guardia Civil en todas sus consecuencias, sin importarle que dar cuenta de la consiguiente pérdida de la brillantez y vistosidad de antaño. La suya se convierte en la descripción estándar para muchos años. Consiste por lo general en la mención de los siguientes elementos, normalmente en el orden siguiente: color dominante del uniforme + color y/o forma de los correajes + color y/o forma del sombrero + (color y/o forma de la capa) + armamento (normalmente colgado al hombro):

The rural police [Guárdias Civiles (sic)] approached in their gray uniforms with shiny yellow belts, cross straps over breasts, patent leather tricorne hats, and the very formidable appearing carbinas (sic) slung non-chalantly across their shoulders. (c.1928:55)

Los viajeros por la España republicana (1931-36) le dan un giro considerable a la imagen visual de la Guardia Civil. En efecto, se siguen centrando en los elementos constantes del uniforme del guardia civil, si bien no tanto ya en el sombrero, que pasa a tener un protagonismo muy reducido en comparación a otras épocas. Tales viajeros insisten durante estos años con evidente frecuencia en la posesión de armamento de fuego, dispuesto a entrar en funcionamiento en cualquier momento o en evidente alarde. También aparece ocasionalmente citada la dependencia del guardia civil al caballo, símbolo éste de atropello y represión. En efecto, en las descripciones del guardia civil pesa ahora más la ostentación de su armamento. Richard and Phyllis Pearsall (Castilian Ochre, 1935) describen muy someramente a un grupo de guardias que ven en Miranda del Ebro. En tan telegráfica descripción no falta la obligada referencia al armamento: 'a bunch of Guardias Civiles with their cloaks, rifles and sandwiches emerged from the station' (1935:12). Charles Graves (Tryptique, 1936) presenta la descripción estandarizada de la uniformi- 
dad del guardia civil en varias ocasiones. Asegura haber reconocido a la Guardia Civil a su llegada a España, a la que nunca había visto antes, gracias a 'their black tricorn helmets' y a 'their bayoneted rifles' (1936:19). En Gijón, entre el posrrevolucionario despliegue de los usuales cuerpos de seguridad una vez aplacada la sublevación de Octubre de 1934, se encuentra naturalmente la Guardia Civil -'with their yellow-handled bayonets' (74)-, presencia que, añade, le proporciona además un cierto toque de colorido militar al paisaje urbano asturiano. Aparte de la obligada referencia a la omnipresencia de la Guardia Civil en un Oviedo recién reconquistado, Matt Marshall, autor de Tramp-Royal in Spain (1935), recuerda además el despliegue y alarde armamentista de la Benemérita. Cada guardia civil portaba una pistola automática y un sable al cinto mientras otros muchos llevaban un rifle a la espalda (1935:7-8). Como puede apreciarse, el sombrero tiene ahora escasa presencia literaria.

Para los viajeros anglófonos por la España republicana el uso del uniforme y el ir armados convierten a los guardias civiles (y por extensión a los guardias de asalto y a otros cuerpos militares o de seguridad) en personajes 'ineptos' ('morons') de la España del momento, ya que, razona Marshall, ninguna persona que se precie de inteligente puede llevar uniforme o llevar armas, o ambas cosas: sólo los niños, los miembros del Ejército y la Marina, los actores, los lunáticos, la realeza y otros irresponsables de tal calibre las portan, y eso se debe, sigue razonando, a que 'no saben lo que hacen' (1935:9). Para el autor de Tramp-Royal in Spain, entre los rasgos distintivos del guardia civil, aparte de los tradicionales (sombrero, capa, etc.), destaca ahora su condición de cuerpo armado. En otra ocasión, cuenta Marshall, un vagabundo suizo y él mismo trataban de evitar ser vistos por lo que parecía una pareja de guardias civiles, y acabaron escondiéndose bajo un puente. Las características sombras de los perseguidores les confirmaron que se trataba de dos miembros de la Guardia Civil. Esas sombras procedían de los sombreros y de las armas que portaban (126). Ahora casi todos, especialmente la tipología conocida como viajeros-vagabundos, tan característicos de la época (Ruiz Mas, 2003), presentan a una Guardia Civil con un prestigio debilitado e impopular, a una Guardia Civil alejada ya de la imagen de cuerpo intocable y ejemplar que llegó a erigirse como modelo a imitar por todos los ejércitos de Europa y América, a una Guardia Civil que abusa del pueblo llano. Abundan las referencias a una Guardia Civil alejada de esa exaltación rayana en la idealización de otras épocas, rozando en ellas en alguna ocasión incluso en el ridículo. Sydney A. Clark narra en Spain on $£ 10$ (1934) con evidente intención humorística cómo unos conejos ensuciaron con sus excrementos a un guardia civil de reluciente sombrero durante un viaje en tren de Alicante a Granada. Naturalmente, los pasajeros que contemplaron la escena disfrutaron de lo lindo (1934:223-24).
En Journeys Between Wars (1938) John Dos Passos no se detiene a describir el uniforme de los guardias civiles, mas emplea otros rasgos para caracterizarlos: si van a pie, destaca sus poblados mostachos y el generoso armamento -'You could shout Viva la Republica right into the moustaches and the Mausers of the Civil Guard without being arrested' (1938:305-6) —; si van a caballo, son los cascos de los caballos los rasgos distintivos del guardia, que no por ello deja de portar algún tipo de arma, en este caso el sable: los manifestantes, dice, gritaban "Live the Republic' as they ran from under the hoofs of the horses and the flailing sabres of the Civil Guards' (305).

En líneas similares están las descripciones que de la Guardia Civil presenta Laurie Lee en As I Walked Out One Midsummer Morning (1969). ${ }^{3}$ Éste se preocupa poco de la descripción del uniforme del guardia y mucho menos de su proverbial vistosidad o de la forma del peculiar sombrero (a estas alturas ya poco novedoso). Para Lee la Guardia Civil se caracteriza por el tamaño y acción de sus monturas y por la posesión de armamento, al igual que hiciera Dos Passos. Tras su desafortunado encuentro con una pareja en una venta cercana a Zamora, Lee dice de los guardias civiles que 'they would suddenly ride down upon you on their sleek black horses, far out in the open country, and crowd around you, all leather and guns (...)' (1969:89). Para Lee la figura del guardia civil se puede estilizar en caballo y arma. Relata además la acción cobardemente represora del Instituto armado (que se repite de boca en boca entre los miembros de los estamentos más humildes), protoganizada por el tamaño y poder destructor de sus monturas: 'Civil Guards on horses the size of elephants riding down the women and children (...)' (156).

Durante varios años más (república, guerra civil y primera década de la posguerra franquista) siguen parcialmente vigentes los elementos del uniforme que tradicionalmente han caracterizado a la Guardia Civil, pero el sombrero sigue teniendo reducida su parcela, quizás por agotamiento literario o por ser un elemento visual más que conocido por el lectorado anglófono y por lo tanto alejado ya a lo que en otras épocas era absoluta novedad. Ahora se incide más en describir su uniforme en su conjunto. Aún hay quien sigue señalando el arcaísmo y pintoresquismo de su atuendo. En Mediterranean Island (c.1949) Lady Margaret Kinloch Sheppard dice de éste que es 'handsome and picturesque', para a continuación añadir que diríase que procede de los tiempos de los Tres Mosqueteros (c.1949:106). Frank Emmott, autor de A Tale of Don Franco in Madrid (1934), contaba que los 'black patent-leather three-cornered hats' de los guardias civiles recordaban a Wellington y a la guerra de la Independencia (1934:29-30). La australiana Nina Murdoch, autora de She Travelled Alone in Spain (1935), lo califica también de 'most picturesque' y lo asemeja al de los carabineros italianos, si bien, aña- 
de, el de éstos es más propio de rufianes que de otra cosa (1935:55-56). Un a la sazón joven Norman Lewis (Spanish Adventure, 1935) emplea para el uniforme de los guardias civiles el calificativo de 'anachronous' (1935:84). En Quest for the Griffon (1938) el naturalista Robert Atkinson, además de los obligados 'picturesque' y 'smart', emplea para su uniforme el calificativo de 'effective', pues consigue asustarle cada vez que alguno de sus portadores aparece por su campamento $-\mathrm{y}$ lo hacen con frecuencia- con la intención de comprobar su identidad y la de sus dos compañeros de investigaciones. Atkinson está convencido de que los guardias sospechan de ellos y tratan de averiguar el que creían 'verdadero' propósito que se escondía tras el estudio de las costumbres del buitre (1938:50).

Pero el sombrero, si bien venido a menos durante los últimos años, no ha llegado a perderse del todo en las descripciones del guardia civil de las épocas republicana y post-republicana. Ahora aparece sobre todo descrito en combinación con otras prendas. Atkinson (1938:50) se refiere al color del uniforme ('olive green'), al color de las bandoleras de los rifles ('bright yellow'), al de las botas ('very shiny black botton-up boots', de aspecto a su juicio escasamente militar), y al del sombrero (entre otros calificativos, 'stiff and black'). Marshall (1936:7-8) describe a los miembros del Cuerpo haciendo referencia a los tres elementos claves de su uniforme, a saber, el color verde de la guerrera y pantalón, las bandoleras amarillas y el característico sombrero negro, descripción que complementa con la obligada alusión al armamento heredada de los viajeros de la década de los treinta:

Moreover, amidst this desert of khaki roamed members of the Guardias Civiles, mostly in twos, conspicuous in their green uniforms, yellow patent leathers, and black gazed hats with the funny turn-ups at the back. And each had an automatic pistol and sword at his belt, while quite a few carried a rifle slung behind. (7-8)

Aunque el sombrero ha venido a menos, independientemente de la ideología o afinidad o no con la Guardia Civil del viajero, éste sigue aludiendo a él acompañándolo también de algún tipo de comentario personal que varía de uno a otro. Murdoch considera que los guardias tienen aspecto siniestro debido precisamente a los sombreros. Los describe de 'queer patent-leather hats, completely brimless in front, but with an affair like a coal-scuttle attached to the back and sides of the crown' (1935:56). Marshall (1936:7) dice que tienen un 'funny turn-up at the back'. A Atkinson (1938:50) y a sus compañeros de exploración les llama la atención lo extraordinariamente surrealista de su forma, que no terminan de 'comprender': rígidas, anchas y angulares alas, a veces horizontales, a veces verticales. De ahí que los califiquen de 'mudguard hats', comparación harto expresiva.
Otros viajeros hay que viajan por la España en guerra o durante la posguerra, ciertamente los menos, viajeros éstos supuestamente afines al bando nacional o neutrales ante el conflicto bélico que desangraba al país (Ruiz Mas, 2008), que pretenden recuperar parte del protagonismo perdido del sombrero del guardia civil con alusiones más abundantes a él, alusiones acompañadas de referencias a las bandoleras y el color verde de sus uniformes. De ahí que Nancy Ford-Inman y Marion L. Nutting (Spinsters in Spain!, 1938) hablen ahora de los guardias civiles como 'the men with the yellow straps and the black hats' (1938:25). Charles Graves (hermano de Robert Graves) concede un absoluto protagonismo al sombrero, mas no por eso se olvida de mencionar 'the usual crossed yellow bandoliers' (1936:102). Lady Sheppard ve a un grupo de guardias pasear a lo largo de un andén. De ellos destaca 'their long carbines, bright yellow bandoliers and shiny black waterproof hats' (c.1949:12).

Incluso tales viajeros siguen haciendo referencias al arcaísmo que rezuman sus sombreros. Lady Sheppard añade que estos 'shiny waterproof hats, turned up sharply at the back' hace que los guardias civiles parezcan tías solteronas del siglo pasado (12). A FordInman y Nutting (1938:25) les atraen los sombreros de los guardias civiles, de los que dicen ser lisos por detrás para que los guardias que los llevan puedan permanecer de pie sobre la pared de los pasillos y así atrapar malhechores sin ser vistos. Sus portadores, dicen las viajeras, parecen figurines abandonados de una representación teatral del 'Mikado' (25). Charles Graves (1936:96), que decía reconocer al guardia civil por su 'black tricorn helmet', es el único viajero del momento que describe el sombrero de la uniformidad de verano. Lo asemeja al de la romántica Legión Extranjera francesa, tan popularizada por Beau Geste de P. C. Wren y luego por el cine: 'Both of them [Guardia Civile (sic)] wore canvas flaps on the backs of their necks, just like the Foreign Legion in a Hollywood film' (96). En plena posguerra franquista V. S. Pritchett dice en The Spanish Temper (1954) estar convencido de que tanto la vistosidad de la vestimenta de los majos de la época de Goya como la de los uniformes militares españoles son reflejo de la extravagancia que caracteriza al pueblo español (1954:55-56).

Los viajeros que llegaron a España con anterioridad al boom turístico de las décadas de los cincuenta y sesenta vuelven a intentar presentar a una Guardia Civil de uniformidad de indudable originalidad y singularidad: 'distinctive', según Robert Henrey en A Journey to Gibraltar (1943:50); pintoresca ('[la Guardia Civil] added a touch of picturesque to the wild surroundings', escribe P. Johnston-Saint en Castanets and Carnations (Castañuelas y Claveles)) (1946:214); o sumamente pintoresca y anticuada, la que más de entre todas las existentes en España, según Churton Fairman en Another Spain 
(1952:15). Y una vez más entra en juego el sabor arcaico que rezuman los tricornios. En este sentido S. F. A. Coles, autor de Spain Everlasting (1945), asegura que la Guardia Civil se creó durante las guerras napoleónicas (sic) para a continuación calificar su sombrero de 'Napoleonic tricorne hat of black oilcloth' (1945:25 y 27). La poetisa Rose Macaulay (Fabled Shore, 1949) considera que el sombrero transporta a la Guardia Civil a otro siglo (1949:21), y Norman Lewis asegura en Voices of the Old Sea (1984) que a primeros del siglo diez y nueve $(1984: 65){ }^{4}$

Una vez finalizada la guerra civil española, los - relativamente escasos - viajeros que visitaron España o regresaron a ella (si habían sido residentes expatriados) se muestran nerviosos de entrar en un país bajo un régimen totalitario. Acostumbran a relatar sus experiencias particulares a su paso por la frontera. Pero ninguno de ellos menciona ya la figura del carabinero, que ha desaparecido para siempre del paisaje español. ${ }^{5} \mathrm{En}$ su lugar aparecen guardias civiles. La uniformidad del guardia civil destinado en Costas y Fronteras se caracterizó a partir de septiembre de 1940 por carecer del sombrero popularmente llamado 'tricornio'; a cambio llevaban gorra de plato (Bueno 1979:72). Ésta puede ser la razón por la que algunos viajeros del momento no se encuentren totalmente seguros de estar viendo a guardias civiles y de ahí que eviten llamarlos como tales. A su llegada y partida por Algeciras Lee observa que tanto en la aduana como en el puerto aparecen 'green-cloaked policemen carrying pistols' y 'greencloaked policemen who leant dozing on their muskets' (A Rose from Winter, 1955:12 and 120). Por el contrario, tierra adentro, no duda en calificar a los guardias civiles como lo que son, 'Civil Guards' (31, 32, 82, 90, 96 y 102). Macaulay observa al atravesar la frontera que separa el Rosellón del Ampurdán que 'the small dark frontier guards' que la resguardan son amables 'guards of green olive coloured uniforms and black and shiny three-cornered hats of another century' (1949:21).

El gran protagonista de la uniformidad de la Guardia Civil de la segunda mitad del siglo XX -años en que da comienzo el conocido como periodo turístico del régimen franquista- vuelve a ser su característico sombrero. Prácticamente todos se detienen en éste, en su original forma y color. La gran mayoría alude a los tres picos de que supuestamente consta. Para ello emplean sobre todo la sempiterna expresión 'a threecornered hat', pero a veces algunas otras tan peculiares como 'tricorne hat', 'tricornis' (sic), 'triangular hat' o incluso 'oddly-shaped shiny black hat with the broad brim turned up at the back'. Suele añadírseles además un toque descriptivo personal: una referencia a su color negro brillante, '(curious) black shiny/shiny black' o 'glistening black'; o al 'patent-leather' o charol de que está recubierto, '(black) oilcloth/patent-leather'. La australiana Frank Clune (pseudónimo de Eleanor Burford)
(1952:52) incide en Castles in Spain (1952) en la extravagancia y teatralidad del sombrero del guardia civil al compararlo con prendas tan peculiares como 'a London bobby's helmet, a guardsman's bearskin, or a Scot kilt' (1952:52). Nina Epton, autora de Grapes and Granite (1956), llega por vía marítima a Vigo. Al bajarse del barco observa el rápido movimiento de los pasajeros en dirección a las barandillas, así como de los Guardias Civiles hacia el muelle, con sus capas color verde oliva y sus relucientes 'tricornes' (1956:7).

En efecto, durante las dos últimas décadas del régimen franquista, que se corresponden con el espectacular desarrollo de la industria española del turismo (1952-1975), la prenda protagonista de las descripciones del vestuario del guardia civil vuelve a ser el sombrero. Si en otras épocas ya lo venía siendo (con sus altibajos), en ésta en que el uniforme del guardia civil se hace más sombrío como consecuencia de la reforma de 1943, el sombrero toma una mayor relevancia aún por su vistosidad o peculiaridad. En contraste a las variadas referencias al vestuario y armamento, el sombrero es la única prenda del uniforme reglamentario que ha permanecido constante (o ha sufrido relativamente escasas modificaciones) desde la creación del Cuerpo. En Image of Spain (1961) James Cleugh lo describe de 'glossy, black, three-cornered hat' (1961:146); Ray Dorien (Venturing Abroad, 1961) de 'oddly-shaped hats with a three-cornered effect' (1961:108); Eric Cleugh, autor de Viva Mallorca (1963), de 'uncomfortable-looking three-cornered black hat' (1963:149); y John Langdon-Davies, en Spain (1971), de 'black, patent-leather three-cornered hat' (1971:32). Su aspecto arcaico y pasado de moda y su inconfundible forma - en esto, poco se ha cambiado- le permiten al viajero y al lector anglosajón remontarse a épocas pasadas. Los viajeros del periodo turístico del régimen franquista (ahora mucho más suave, permisivo y comprensivo con el visitante de fuera), con frecuencia siguen vinculándolo a la Guerra de la Independencia, a la época de Goya o de Napoleón, evidentes anacronismos convertidos en auténticos clichés, o más acertadamente, al reinado de Isabel II. William Sansom por ejemplo, autor de Away to it All (1964), lo califica de 'Napoleonic hard leather helmet' (1964:163); Madeleine Duke, autora de Beyond the PiIlars of Hercules (1957:68), de 'Napoleonic hats'; Epton (1956:7) lo califica de 'shiny tricornes that belong sartorially to the days of Goya and the Peninsular War'. Incluso en sí la referencia a la condición de 'three-cornered hat' que numerosos viajeros emplean para describirlo lleva ya implícita la connotación de sombrero antiguo, más propio de la España tradicional y eterna que de la moderna. La degradante tendencia de considerarlo un sombrero ridículo propio de circos y operetas de barrio se ha reducido considerablemente, mas no ha desaparecido totalmente. En Spain and her People (1961) Dorothy Loder los describe aún como 'three-cornered patent-leather hats belonging to the chorus of an ope- 
ra' (1961:99). Y R.A.N. Dixon (Spanish Rhapsody, 1955) los califica de 'shiny black patent-leather three-cornered caps that you would think could be encountered nowhere but in musical comedy' (1955:57).

El reglamento de 1943 impuso en la uniformidad del guardia civil un espectacular sombrero de gala, hecho del que da debida cuenta W. T. Blake en Spanish Journey; or, Springtime in Spain (1957), que tiene oportunidad de contemplarlo en las fiestas de Valencia y de Tarragona. Lo califica de 'gold hat' debido al 'gold braid' que lo recubre (1957:161 y 164). Shirley Deane, también autora de Tomorrow is Mañana (1957), no lo describe, pero da muestra de su admiración puramente visual por él al verlo usado precisamente por el antipático teniente de la Guardia Civil en uniforme de gala que intentó hacer lo posible por impedir - sin éxito- que viajase a Málaga para ver a Franco en persona (1957:107). La referencia más curiosa al uniforme de gala la hace Chapman Mortimer en Here in Spain (1955): el viajero cree estar ante un guardia civil de alto empleo cuando en realidad se trata de un agente vestido de uniforme de gala (1955:310).

Con la excepción del sexenio republicano, parte de la guerra civil y primeros años de la posguerra, la peculiar forma del sombrero del guardia civil ha suscitado casi siempre la curiosidad del viajero. El sentido pragmático que caracteriza al anglosajón le lleva a interesarse por el origen de las alas a ambos lados y su verdadera utilidad. En efecto, cuando un extranjero entabla cierta confianza en la conversación con algún miembro del Instituto armado, no desaprovecha la ocasión para plantearle la cuestión, tal y como ya hicieran otros viajeros de épocas anteriores. Un guardia civil se monta en el automóvil de Blake (1957:77-88). A la pregunta de la esposa de Blake, el militar dice ignorar el porqué de tan peculiar forma, pero alaba su conveniencia en los días de lluvia, pues el charol que lo recubre repele el agua; es más, añade, en verano el sombrero admite visera y cogotera como protección a la intensa luz y el calor abrasador del estío español. Un culto sargento de la Guardia Civil con el que Robert Hugill (I Travelled in Spain, c.1967) entabla conversación durante el trayecto ferroviario de Alicante a Granada le informa, entre otros aspectos curiosos e interesantes del Cuerpo, sobre el origen de la transformación del antiguo sombrero de tres picos a su forma actual: la de conmemorar una heroica defensa que protagonizaron los guardias civiles frente a los carlistas (c.1967:128). Langdon-Davies (1971:32) asegura que la forma del sombrero reglamentario no es caprichosa: le permite a su portador 'lean up against walls or trees'.

El característico sombrero del guardia civil, escribe Colin Simpson, autor de Take Me to Spain (1963:57), debe su rareza a la forma tan peculiar que tiene. Pero no todos los viajeros se ponen de acuerdo en el modo de describir su característico contorno. En Viva Ma-
Ilorca (1963) Eric Cleugh lo cree resultado de la simplificación del sombrero de tres picos en un solo pico (1963:149). J. M. Scott (From Sea to Ocean, 1969) lo califica de 'funny' sin entrar en detalles (1969:57); el poeta y novelista Robert Graves de 'peaked hat' en Majorca Observed (1965:33); Michael Perceval (The Spaniards: How They Live and Work, 1969) de 'tricorne headgear' (1969:32), y Peter de Polnay (Peninsular Paradox, 1958) simplemente de 'tricornio' (1958:157). Los hay que se esfuerzan en dar una descripción lo más precisa posible. Según Hugill (c.1967:7-8), el sombrero del guardia civil es 'a patent-leather hat turned up at the back to form two wings'; para Bernard Newman, autor de Spain on a Shoestring (1957), este 'queer hat' consiste en 'a pill-box of shiny black leather, with flat backs -rather like a schoolgirl's straw hat, enamelled black and with the brims turned up' (1957:52). Resulta evidente que desde sus primeras descripciones, la visión del sombrero a ojos de los viajeros de habla inglesa ha evolucionado bien poco con el paso del tiempo.

Otro rasgo propio del sombrero del guardia civil es el material del que está recubierto, 'patent-leather' (charol), material popularizado en el mundo anglosajón gracias a las numerosas traducciones al inglés del 'Romance de la Guardia Civil Española' de Federico García Lorca. 'Alma de charol' (verso 7) ('patent-leather soul') ha resultado ser una imagen muy afortunada ampliamente usada para referirse al Cuerpo. La identificación del charol con la imagen del guardia civil se hace evidente cuando el novelista y viajero norteamericano James Michener escribe en Iberia (1968) que los intelectuales de la Izquierda suelen aludir a la Guardia Civil como 'those patent-leather men with patent-leather souls' (1968:66), particular adaptación del citado verso lorquiano. Mortimer califica tan características prendas de 'waxed hats' (1955:310), imagen también de ciertas reminiscencias lorquianas ('manchas de tinta y de cera', verso 4).

\section{CONCLUSIÓN}

Tras el extenso recorrido por el género de relatos de viajes por la España del siglo XX desde la perspectiva de la visión y consiguiente descripción que del tricornio del guardia civil han incluido numerosos autores en sus obras, cabe resaltar una serie de conclusiones. La imagen colectiva que de la Guardia Civil guarda la comunidad angloparlante desde el siglo XIX hasta la actualidad -entendida ésta como las últimas décadas del siglo XX e incluso primera década del XXI- es consecuencia de la imagen literaria transmitida en los relatos de viajeros de habla inglesa por España en un periodo que abarca más de siglo y medio. En efecto la Guardia Civil es vista y descrita por éstos como un cuerpo omnipresente en nuestros lares, recipiente de una particular combinación muy española de firmeza y amabilidad, eficiencia y ocasional brutalidad en sus métodos; es un cuerpo 
vinculado a los poderes fácticos, al status quo y más recientemente al régimen franquista, con el que pronto sintonizó y al que contribuyó a mantener en el poder; esto le convierte en un cuerpo reticente al nuevo régimen democrático. Prueba del trabajo que le ha costado aceptarlo es la frecuente alusión que se hace en los últimos relatos de viajes al teniente coronel Tejero como símbolo del inmovilismo de parte del Ejército y la Guardia Civil. Es además erróneamente considerado un cuerpo 'paramilitar', ostentosamente armado - hecho que para muchos viajeros anglófonos constituye toda una provocación-, de vistoso uniforme arcaizante, especialmente por su peculiar sombrero, y enemigo acérrimo del bandolero, del contrabandista, del carlista, del republicano, del mendigo y del vagabundo, del gitano, del guerrillero antifranquista, del 'guapo' o matón, del anarquista, del comunista, del socialista, del nacionalista, del terrorista y de los estamentos sociales más humildes, o sea, de casi todos los tipos humanos que han venido suscitando simpatías a una parte considerable de los viajeros de habla inglesa en diversas épocas de la historia de España.
Mas en lo que se refiere al 'tricornio' como símbolo literario de un cuerpo tan peculiar y representativo del paisaje humano, histórico y de la realidad del día a día de la España contemporánea el viajero anglófono presenta una serie de rasgos que, a título de conclusión, podrían resumirse de la siguiente forma. Resultan harta numerosas las ocasiones en que se califica el característico sombrero del guardia civil como 'a three-cornered hat', o 'a cocked hat', o en que se atribuye su peculiar forma a la conveniencia de sus portadores a pegarse a la pared y atrapar mejor a los malhechores, o en que se menciona su parentesco - a todas luces disparatadocon la guerra de Independencia o Napoleón o Goya; o a la Gendarmería francesa, vinculación sin embargo mejor encaminada; o el arcaísmo de su uniforme, que se remonta a sus orígenes en el siglo XIX; o su condición de 'picturesque', o su parecido con el de los monigotes de feria u operetas. La presencia constante de esta característica prenda en el paisaje literario de los viajeros de habla inglesa - prueba inequívoca de la omnipresencia de sus portadores - resulta a todas luces innegable en la España tal y como la conocemos hoy en día.

\section{NOTAS}

1 Durante la guerra civil española el bando republicano la convirtió durante unos meses en Guardia Nacional Republicana, para finalmente integrarla en un cuerpo llamado de Seguridad. En la parte inicialmente controlada por las tropas del general Franco la Guardia $\mathrm{Ci}$ vil siguió intacta, ejerciendo sobre todo labores de retaguardia con los cometidos que tradicionalmente le han sido propios. Luego estamos en condiciones de afirmar que ésta no ha desaparecido del paisaje español durante sus casi 170 años de existencia.

\section{BIBLIOGRAFÍA}

Alburquerque-García, Luis (2011). "El 'relato de viajes': hitos y formas en la evolución del género". Revista de Literatura, enero-junio, vol. LXXIII, no 145 , págs. 15-34.

Atkinson, Robert (1938). Quest for the Griffon. On the Author's Experiences while photographing the Griffon Vulture in Spain. London, Toronto: William Heinemann.

Bailey, Vernon Howe (1928). Little Known Towns of Spain. New York: William He-
2 Aunque Brenan publicaba South from Granada en 1957, la obra se refiere en su mayor parte a su estancia como residente en la Alpujarra granadina durante la década de los 20 (en plena dictadura de Primo de Rivera). Pero en 1957 España se encontraba bajo el control de otra dictadura, la del general Franco. La alusión va pues dirigida a ambos regímenes. Brenan es uno de los viajeros, hispanistas e historiadores ingleses más relevantes del siglo XX.

3 Aunque publicado en 1969, el relato de Lee narra sus experiencias como joven

Iburn Inc.; London: B. T. Batsford, c. 1928.

Blake, W. T. (1957). Spanish Journey or Springtime in Spain. London: Alvin Redman.

Brenan, Gerald (1957). South from Granada. London: Hamish Hamilton.

Bueno, José María (1979). Uniformes Militares Españoles: La Guardia Civil 18441978, Málaga.

Clark, Sydney A. (1934). Spain on $f 10$. London: Ivor Nicholson and Watson. viajero aspirante a escritor por una España republicana cercana cronológicamente al estallido de la guerra civil.

4 Norman Lewis publicó en 1984 Voices of the Old Sea, obra en la que narraba sus experiencias en un pueblecito costerio catalán, Farol, durante los duros años de la posguerra franquista.

5 El cuerpo de Carabineros, creado en 1827 por el Marqués de Rodil, era fusionado con la Guardia Civil en 1940. Franco castigaba así la adhesión mayoritaria de los carabineros a la República durante la guerra civil.

Cleugh, Eric (1963). Viva Mallorca. Yesterday and Today in the Balearic Islands. London: Cassell.

Cleugh, James (1961). Image of Spain. London, Toronto, Wellington, Sydney: George G. Harrap.

Clune, Frank (1952). Castles in Spain. A Flying Trip from Australia to Europe with some Quixotical Peregrinations in the Iberian Peninsula in Quest of Facts. Sydney, London: Angus and Robertson. 
Colam, Lance (1957). Southern Spain on f25. London: Frederick Muller.

Coles, S. F. A. (1945). Spain Everlasting. London: Hollis \& Carter.

Daly, Maureen (1960). Spanish Roundabout. New York: Dodd, Mead \& Company.

Deane, Shirley (1957). Tomorrow is Mañana. An Andalusian Village. London: John Murray.

Dixon, R. A. N. (1955). Spanish Rhapsody. London: Robert Hale.

Dorien, Ray (1961). Venturing Abroad. Majorca and Ibiza. London: Christopher Johnson.

Dos Passos, John (1938). Journeys between Wars. London: Constable.

Duke, Madelaine (1957). Beyond the Pillars of Hercules. A Spanish Journey. London: Evans Brothers.

Emmott, Frank (1934). The Tale of Don Franco in Madrid. A True Account of What He Saw and Did. Halifax: F. King \& Sons.

Epton, Nina (1956). Grapes and Granite. London: Cassell.

Fairman, Churton (1952). Another Spain. London: Museum Press.

Ford-Inman, Nancy and Marion L. Nutting (1938). Spinsters in Spain! London: Heath Cranton.

Freeston, Charles L. (1930). The Roads of Spain. A 5000 Miles' Journey in the New Touring Paradise. London: Humphrey Toulmin.

Graves, Charles (1936). Trip-Tyque. London: Ivor Nicholson and Watson.

Graves, Robert \& Paul Hogarth (1965). Majorca Observed. London: Cassell.

Hawkes, C. P. (1926). Mauresques. With some Basque and Spanish Cameos. London: Methuen.

Henrey, Robert (1934). A Journey to Gibraltar. London: J. M. Dent \& Sons.

Hugill, Robert (1967). I Travelled through Spain. Wimbledon: Vernon \& Yates, c.1967.

Johnston, Sir Harry and Dr. L. Haden Guest, eds. (1930). "'Spain", The Outline of the
World To-day. 3 Vols. London: George Newnes, c.1930.

Johnston-Saint, P. (1946). Castanets and Carnations (Castañuelas y Claveles). London: Heath Cranton.

Langdon-Davies, John (1971). Spain. London: B. T. Batsford.

Lee, Laurie (1969). As I Walked Out One Midsummer Morning. London: André Deutsch.

Lee, Laurie (1955). A Rose for Winter Travels in Andalusia. London: André Deutsch.

Lewis, Norman (1984). Voices of the Old Sea. London: Hamish Hamilton.

Lewis, Norman (1935). Spanish Adventure. London: Victor Gollancz.

Loder, Dorothy (1961). Spain and her People. London: Lutterworth Press.

Macaulay, Rose (1949). Fabled Shore. From the Pyrenees to Portugal. London: Hamish Hamilton.

Marshall, Matt (1935). Tramp-Royal in Spain. Edinburgh and London: WM. Blackwood \& Sons.

McClymont, James R. (1921). A Scot in Spain. Glasgow: Maclehouse, Jackson and Co., Publishers to the University.

Michener, James A. (1968). Iberia. Spanish Travels and Revelations. New York: Fawcett Crest.

Moore, Thomas Ewing (1927). In the Heart of Spain. New York: The Universal Knowledge Foundation.

Mortimer, Chapman (1955). Here in Spain. London: The Cresset Press.

Murdoch, Nina (1935). She Travelled Alone in Spain. London, Bombay, Sydney: George G. Harrap.

Newell, Lieut.-Colonel H. A. (1922). Footprints in Spain. London: Methuen.

Newman, Bernard (1957). Spain on a Shoestring. London: Herbert Jenkins.

Pearsall, Richard and Phyllis (1935). Castilian Ochre. London: John Murray.

Perceval, Michael (1969). The Spaniards: How They Live and Work. Newton Abbot: David \& Charles.
Phillips, Henry Albert (1931). Meet the Spaniards. In which Spain is Seen Primarily through the Life of the Spanish People. Philadelphia and London: J. B. Lippincott.

Polnay, Peter de (1958). Peninsular Paradox. London: MacGibbon \& Kee.

Pritchett, V. S. (1954). The Spanish Temper London: The Hogarth Press.

Richardson, Captain Leslie (1928). Things Seen in the Pyrenees, French \& Spanish. A Description of an Interesting Frontier Land, its Wonderful Scenery, Romantic Associations, the Attractions of its Fashionable Resorts, \& the Charms of its more Secluded Retreats. London: Seeley, Service \& Co.

Riley, Alice C. D. (1931). Skimming Spain in Five Weeks by Motor. Los Angeles: Saturday Night Publishing Company.

Ruiz Mas, José (2003). Libros de viajes en lengua inglesa por la España del siglo $X X$. Granada: Grupo Editorial Universitario y UNED de Jaén.

Ruiz Mas, José (2005). La Guardia Civil en los libros de viajes en lengua inglesa. Málaga: Servicio de Publicaciones de la Universidad de Málaga.

Ruiz Mas, José (2008). La Guerra Civil española vista por los viajeros y los historiadores de habla inglesa. Granada: Editorial Universidad de Granada.

Ruiz Mas, José (2010). Guardias civiles, bandoleros, gitanos, guerrilleros, contrabandistas, carabineros y turistas en la literatura inglesa contemporánea (1844-1994). Bern, Berlin, Bruxelles, Frankfurt am Main, New York, Oxford, Wien: Peter Lang.

Sansom, William (1964). Away to It All. London: The Hogarth Press.

Scott, J. M. (1969). From Sea to Ocean. Walking along the Pyrenees. London: Geoffrey Bles.

Sheppard, Lady Margaret Kinlock (c. 1949). Mediterranean Island. London, New York, Melbourne, Sydney, Cape Town: Skeffington and Son.

Simpson, Colin (1963). Take Me to Spain. Including Majorca and with a Sampling of Portugal. London, Sydney, Melbourne, Wellington: Angus \& Robertson. 